was $37.37 \pm 13.96$ years old, the average duration was 6.7 \pm 5.8 years. Among them, 310 patients were in glucocorticoid maintenance stage. $61 \%$ of patients received the medium dosage $(30-60 \mathrm{mg} / \mathrm{d})$ as the initial treatment dosage of glucocorticoid. However, patients receiving different initial dosage had no discrepancy on glucocorticoid in the maintenance therapy. In the maintenance stage, $51 \%$ of patients received $2.5-5 \mathrm{mg} /$ $\mathrm{d}$ prednisone, while the dosage of $5-10 \mathrm{mg} / \mathrm{d}$ could maintain for a longer time. Patients with internal organs involvement had a higher tendency to receive $60-100 \mathrm{mg} / \mathrm{d}$ or pulse-dose therapy in the initial treatment, nevertheless there had no difference on the dosage of glucocorticoid in the maintenance stage. Among the 400 patients, 62 patients had glucocorticoid withdrawal, including 17 patients due to disease remission (17/400, 4.25\%), 44 patients due to self-withdrawal $(44 / 400$, $11 \%)$ and one patient due to adverse reaction $(1 / 400,0.25 \%)$. Conclusions In China, the medium dosage of glucocorticoid is the most common choice in the initial treatment of SLE patients, and the dosage of $2.5-5 \mathrm{mg} / \mathrm{d}$ was most common in maintenance stage. Currently, the proportion of glucocorticoid withdrawal remains low after SLE patients achieving the remission.

\section{CHARACTERISATION OF PATIENTS WITH SYSTEMIC LUPUS ERYTHEMATOSUS WHO WERE ADMITTED TO A UNIVERSITY HOSPITAL IN BOGOTA-COLOMBIA BETWEEN 2008 AND 2016}

\begin{abstract}
1J Aponte, ${ }^{1} \mathrm{~A}$ Cartagena, ${ }^{2} \mathrm{~J}$ Carrizosa, ${ }^{3} \mathrm{~A}$ Sanchez, MT Ospina ${ }^{4},{ }^{5} \mathrm{C}$ Gamboa, ${ }^{6} \mathrm{R}$ Cervera. ${ }^{1}$ Hospital Universitario de la Samaritana - Universidad de La Sabana, Medicina Interna, Bogota, Colombia; ${ }^{2}$ Universidad del Rosario, Cuidado critico, Bogota, Colombia; ${ }^{3}$ Hospital universitario de la samaritana, Medicina interna - Cuidado intensivo, Bogota, Colombia; ${ }^{4}$ Hospital universitario de la samaritana, Cuidado critico, Bogota, Colombia; ${ }^{5}$ Universidad de la Sabana, Medicina - Pregrado, Bogota, Colombia; ${ }^{6}$ Senior y Jefe del Servicio de Enfermedades Autoinmunes del Hospital Clínic de Barcelona, Enfermedades autoinmunes, Madrid, Spain
\end{abstract}

\subsection{6/lupus-2017-000215.388}

Background and aims Systemic lupus erythematosus is one of the most prevalent autoimmune disease, and it is associated with many complications, morbidity and mortality. The mortality in these patients is related with immune activity, infectious complication and a direct effect of pharmacology therapy. All these factors result in target organ injury.

Methods A retrospective-descriptive study of patients admitted to the intensive care unit of the Hospital Universitario de la Samaritana between 2008 and 2016 in the city of Bogota was performed. The aim of this study was to characterise the clinical, demographic and paraclinical features of patients with systemic lupus erythematosus.

Results 56 cases were collected, most of the patients were relatively young with an average age of 40.7 years $(S D \pm 17.7 \mathrm{y} /$ o). These people were mainly female (71\% vs $29 \%)$, the immune activity was measure with score SELENA SLEDAI with average $16.5(\mathrm{SD} \pm 4.3)$, in the analysis of target organ $69 \%$ of patient had lupus nephritis, $42 \%$ haematological commitment, 28\% lung injury, $17 \%$ neurological complications and 48.25 had infectious process. When it comes to the days of mechanical ventilation, the average was 9 (SD \pm 10 ), days of ICU stay 13.7 (SD \pm 14.3 ) and finally the mortality was $39.2 \%$.

Conclusions At ICU of our hospital, the prevalence of patients with systemic lupus erythematosus tends to be more frequent than in other series reported, this information may be useful in future studies and can also reduce complications and mortality in this population.

\section{CLINICAL FEATURES OF SEVERE THROMBOCYTOPENIA IN CONNECTIVE TISSUE DISEASES: A REVIEW OF 131 CASES}

W Zhang*. Jiangning Hospital affiliated to Nanjing Medical University, Rheumatology, Nanjing, China

\subsection{6/lupus-2017-000215.389}

Background and aims To examine the clinical features, prognosis and response to treatments for severe thrombocytopenia in patients with connective tissue diseases (CTDs).

Methods The data of 131 CTDs inpatients with severe thrombocytopenia were reviewed. Severe thrombocytopenia was defined as blood platelet counts (BPC) under $20000 / \mathrm{mm}^{3}$, and patients were divided into 3 groups according to BPC at discharge: no response (NR), partial response (PR), complete remission (CR). The differences of clinical features, treatments, and prognosis were analysed.

Results Of the 131 patients, 70 cases were diagnosed as primary Sjögren's Syndrome (pSS), 53 cases as SLE and 8 cases as other CTDs. $88.6 \%$ of them were female and the mean age of SLE patients was younger than pSS and other CTDs patients. The bleeding severity was negatively correlated with patients' lowest BPC during hospitalisation. BPC at discharge was positively correlated with BPC during follow-up. There was no significant difference in basal data among NR, PR and CR group except serum IgG level that was lower in NR group than PR and CR group. The treatments were identical among the 3 groups, except that the use of IVIG was more frequently in CR group than the other 2 groups. Mortality in patients with partial or complete remission was significantly lower than in those without remission.

Conclusions Severe thrombocytopenia is more common in SLE and pSS patients than in other CTDs and high IgG level may predict a better efficacy. IVIG is helpful to achieve a full response and those unresponsive to the treatments at discharge have poor outcome.

\section{Pregnancy: the mother and the child}

\section{OUTCOMES OF PREGNANCY OF KYRGYZ SLE PATIENTS}

${ }^{1} \mathrm{G}$ Koilubaeva, ${ }^{1} \mathrm{G}$ Koilubaeva*, ${ }^{2} \mathrm{R}$ Tatiyana, ${ }^{2} \mathrm{~A}$ Elena, ${ }^{2} \mathrm{~S}$ Sergei, ${ }^{2} \mathrm{~N}$ Evgeniy, ${ }^{1} \mathrm{D}$ Ainagul, ${ }^{1} \mathrm{E}$ Venera, ${ }^{1} \mathrm{~K}$ Elmira. ${ }^{1}$ National Centre of Cardiology and Internal Medicine, Rheumatology, Bishkek, Kyrgyzstan; ${ }^{2}$ Institute of Rheumatology at Russian Academy of Medical Science, Rheumatology, Moscow, Russia

\subsection{6/lupus-2017-000215.390}

Background and aims To study outcomes of pregnancy of Kyrgyz SLE patients.

Methods Included 75 patients with SLE passed the examination in the clinic NCCIM from 2001 to 2011. Age of women was $30[20 ; 40]$ years old, duration of the disease on the 1 st point-3 $[0.8 ; 40]$ years. Assessed disease activity by SLEDAI $2 \mathrm{~K} 1$ st point and the end point after an average of 6.38 \pm 3.48 years. 
Results Evaluation of outcomes of gestation showed that in 9 cases, there has been a termination of pregnancy in the $1 \mathrm{st}$ trimester due to the high activity of SLE. They were mostly women with active lupus nephritis (7), one case with massive exudative pericarditis and one patient with lupus hepatitis. Pregnancy in 6 women on the background of a moderate (2) and minimal disease activity (4), with a primary skin (4) and articular syndrome (1), with the trace proteinuria (1) completed delivery of healthy children in the gestation of 36 to 38 weeks. In 3 of them - by Caesarean section, in 3 others it was delivery was vaginally at 39 weeks gestation. 27 did not have children because of the risk of adverse effects of pregnancy on the course of the disease; the other 27 women had children prior to the development of SLE.

Conclusions Half of the women (52\%) was not able to have children due to the high risk of adverse effects of pregnancy on the course of the disease, with active SLE caused the interruption of pregnancy in $13 \%$ of patients.

\section{PREGNANCY OUTCOME OF SYSTEMIC LUPUS ERYTHEMATOSUS PATIENTS IN CIPTO MANGUNKUSUMO NATIONAL REFERRAL HOSPITAL, JAKARTA, INDONESIA}

SA Kusumo Wibowo*, A Ariane, SP Hidayat, EJ Yunasan, B Setiyohadi, R Hidayat, S Sarmidi, Z Albar, H Isbagio. RSCM, Division of Rheumatology- Department of Internal Medicine, Central Jakarta, Indonesia

\subsection{6/lupus-2017-000215.391}

Background and aims Systemic Lupus Erythematosus (SLE) is a multisystem autoimmune disease which commonly affects women of childbearing age. There were reports of the adverse pregnancy outcome in SLE patients but data from Indonesia was still lacking. The objectives of this study were to analyse the outcome of pregnancy in SLE patients and to identify the factors associated with pregnancy outcome.

Methods This was a retrospective study of pregnant SLE patients in Indonesian national referral hospital from 20122015. Medical records of all pregnant SLE patients who gave birth in our centre during that period were reviewed. Independent variables were previous and current nephritis, history of adverse pregnancy outcome, pre-pregnancy hypertension, and disease control during pregnancy. Dependent variables were maternal complications (ICU admission, SLE flare, hypertension/eclampsia/preeclampsia, and death) and fetal/neonatal complications (low birth weight, oligohydramnios, and abortion/fetal death/neonatal death).

Results There were 32 pregnancies of which 13 were first pregnancies. Preterm deliveries were observed in $37.5 \%$ patients and $66.7 \%$ patients were delivered by C-section. Eight patients (25\%) experienced maternal complications with 1 patient died due to heart failure. There were $71.9 \%$ pregnancies with fetal/neonatal complications and among those, low birth weight was the main complication (39.1\%). There were significant association between disease control during pregnancy and maternal complications $(\mathrm{p}=0.029)$ and between history of adverse pregnancy outcome and fetal/neonatal complications $(p=0.005)$.

Conclusions The rate of maternal and fetal/neonatal complications was high. Pregnant SLE patients need to be monitored closely especially those with uncontrolled disease during pregnancy and history of adverse pregnancy outcome.

\section{SAFETY AND EFFICACY OF MODIFIED-RELEASE PREDNISONE IN SYSTEMIC LUPUS ERYTHEMATOUS PREGNANCIES: AN IMPLEMENTED COHORT}

${ }^{1} \mathrm{M}$ Meroni ${ }^{*},{ }^{1} \mathrm{M}$ Limonta, ${ }^{2} \mathrm{~V}$ Ramoni, ${ }^{3} \mathrm{M}$ Cutolo. 'ASST Papa Giovanni XXIII, Rheumatology, Bergamo, Italy; ${ }^{2}$ RCCS San Matteo Hospital Foundation- University of Pavia, Division of Rheumatology, Pavia, Italy; ${ }^{3}$ University of Genova- Genova-, Research Laboratory and Academic Division of Clinical Rheumatology- Department of Internal Medicineltaly, Genoa, Italy

\subsection{6/lupus-2017-000215.392}

Background and aims SLE often affects women of childbearing age. Prednisone is safely used during pregnancy. Modifiedrelease prednisone $(\mathrm{MRP})$ is a treatment option, but no data exist regarding gestation: we aimed to compare its safety and effectiveness to the immediate release formulation (IRP) in SLE pregnancies.

Methods During a 5 year period, we enrolled 18 SLE female patients experiencing a pregnancy. Nine (cases), taking lowdose MRP (5 to $7.5 \mathrm{mg} /$ daily) as a baseline treatment, were matched to 9 controls (same age and disease duration), taking the same prednisone dose in the IR formulation. Pregnancy outcome; SLEDAI/SLEPDAI; patient's VAS and need of treatment changes were assessed at baseline, during pregnancy and at postpartum.

Results SLEDAI at baseline was $2 \pm 0.1$ among MPR and 2 \pm 0.3 among IR women; SLEPDAI, $3 \pm 0.9$ and $3 \pm 0.2$ (both, $\mathrm{p}=\mathrm{ns})$. No major perinatal complications were detected. Preterm births, caesarean section rates, newborns weight and APGAR scores did not differ between the two subpopulations (all, $\mathrm{p}=\mathrm{ns}$ ). SLEDAI at postpartum was $3.8 \pm 0.6$ in MRP subjects and $5.4 \pm 0.4$ in IR $(\mathrm{p}<0.05)$. Patients VAS evaluation (MRP vs IR) was, respectively, $30 \pm 4$ and $20 \pm 9$ at baseline $(\mathrm{p}=\mathrm{ns}) ; 30 \pm 6$ and $48 \pm 7$ during pregnancy $(\mathrm{p}<0.05)$ and 31 \pm 3 and $52 \pm 9$ at postpartum $(\mathrm{p}<0.05)$. Prednisone regimen changes (add-on strategy), the observed rates involved 1/9 (MRP) and 5/9 (IR) women $(\mathrm{p}<0.05)$.

Conclusions Among IR patients, activity was significantly higher during postpartum and treatment had to be increased. VAS was significantly different (higher among IR), both during pregnancy and postpartum. MRP seems to be as safe, but more effective, in comparison the IR, during pregnancy of SLE women.

\section{PREGNANCY ASSOCIATED WITH SYSTEMIC LUPUS ERYTHEMATOSUS IN OUR HOSPITAL}

${ }^{1} \mathrm{M}$ Mukai ${ }^{*},{ }^{1} \mathrm{M}$ Kondo, ${ }^{1} \mathrm{H}$ Kanaoka, ${ }^{2} \mathrm{~K}$ Okukyama. 'Sapporo City General Hospital, Division of Rheumatology and Clinical Immunology- Department of Medicine, Sapporo, Japan; ${ }^{2}$ Sapporo City General Hospital, Department of Obstetrics, Sapporo, Japan

\subsection{6/lupus-2017-000215.393}

Background and aims To evaluate the course of pregnancy associated with systemic lupus erythematosus (SLE).

Methods We picked up retrospectively the patients with SLE who was in pregnant. We studied the courses of pregnancy, the results of delivery, and the states of babies.

Results We found 35 courses of pregnancy associated with SLE. Twenty-six patients with SLE experienced pregnancy. Thirty-six babies were born through 35 courses of pregnancy (including a pair of twin). Early deliveries were found in 5 cases (including a pair of twin). Low weight born were found 